\title{
Cluster-Based Context-Aware Routing Protocol for Mobile Environments
}

\author{
Ahmed. A. A. Gad-ElRab \\ Department of Mathematics \\ Faculty of Science \\ Al-Azhar University-Cairo,Egypt
}

\author{
T. A. A. Alzohairy \\ Department of Mathematics \\ Faculty of Science \\ Al-Azhar University-Cairo,Egypt
}

\author{
Almohammady S. Alsharkawy \\ Department of Mathematics \\ Faculty of Science \\ Al-Azhar University-Cairo,Egypt
}

\begin{abstract}
Mobile environment has many issues due to mobility, energy limitations and status changing over time. Routing method is an important issue and has a significant impact in mobile networks, whereas selecting the optimum routing path will reduce the wasting in network resources, reduce network overhead and increase network reliability and lifetime. To decide which path will achieve the networks objectives, we need to construct a new routing algorithm that uses context attributes of a mobile device such as available bandwidth, residual energy, connection number and mobility value. In this paper, we propose a new mobile nodes ranking scheme based on the combination of two multi-criteria decision making approaches, the analytic hierarchy process (AHP) and the technique for order performance by similarity to ideal solution (TOPSIS) in Fuzzy environments. The Fuzzy AHP is used to analyze the structure of the clusterhead selection problem and to determine weights of the criteria, while the Fuzzy TOPSIS method is used to obtain the final mobile node ranking value. By basing on node ranking, we propose a new cluster based routing algorithm select the optimal clusterheads and the best routing path. Our simulation results show that the proposed method increases the network accuracy and lifetime and reduces network overhead.
\end{abstract}

Keywords-Clustering; Context; FMCDM; Mobile and Routing

\section{INTRODUCTION}

Many mobile systems utilize the mobile device context such as current location, residual energy, time and user's activity to obtain the best services to the mobile user. The key objective of these systems is to significantly simplify computing devices usage by realizing the changing in entities status and the surrounding environments. Context-aware systems use the contextual information to clarify the current situation and adapt mobile systems to be suitable for both user and device requirements.

Exchanging data between mobile nodes in the network is one of the basic challenges in this environment. Utilizing a context in mobile devices is receiving considerable attention to meet these challenges. In context-aware systems, mobile applications can use the contextual information such as user's location, day time, nearby people and devices and user's activity in useful way to solve many mobile issues. One of the most important issues in mobile computing is how to evaluate mobile device. Evaluating mobile device using user information, device information and environmental information is very helpful in many mobile applications such as data management and routing data in mobile networks. So, we can rely on the rank values to use the highest performance mobile devices to send data to other nodes in the network. This method will keep most of mobile resources as energy and will increase network lifetime. Using the context in routing data through the network paths will achieve a high accuracy mobile network, and will reduce network overhead. Routing data using cluster methods allows fast connection, topology management, better routing, improves network lifetime, routing delay, bandwidth consumption, and throughput.

The main objective of this research is to introduce a systematic evaluation model to help the actors in mobile computing for evaluating and selecting the optimal mobile node among a set of available alternatives (mobile nodes). Evaluating mobile node based on context is a multi-criteria decision making problem (DM), where many context attributes should be considered in the decision-making. $D M$ processes involve a series of steps: identifying the problems, constructing the preferences, evaluating the alternatives and determining the best alternatives. $D M$ is extremely intuitive when considering single criterion problems, since we only need to specify the alternative with the highest preference rating. However, when $D M$ method evaluates alternatives with multiple criteria (context attributes), many problems will arise in the evaluation process such as criteria weights, preference dependence, and conflicts among criteria. These problems need to be overcome by more sophisticated methods. So, network clustering which is based on multi-criteria will achieve a high performance routing method in mobile environments.

Fuzzy decision making is a method to solve the complex $D M$ problems in a fuzzy environment. This method can deal with the problem of evaluation and selection. In the real world, linguistic variable is used by human beings to make decisions. Classical $D M$ method works only with exact and ordinary data without qualitative data. This research will use the linguistic variable to express reasonably situation that difficult to define such as available bandwidth, residual energy and device mobility factor, and then select the best alternatives for data management in the mobile environment using a cluster based routing protocol.

In this paper, we propose a new ranking scheme for mobile nodes that is based on the combination of two multi-criteria decision making approaches, the analytic hierarchy process (AHP) and the technique for order performance by similarity to ideal solution (TOPSIS) in Fuzzy environments. The Fuzzy AHP is used to analyze the structure of the clusterhead selection problem and to determine weights of the criteria, 
while the Fuzzy TOPSIS method is used to obtain final mobile node ranking. Finally, based on the node ranking value, we propose a new cluster based routing algorithm for selecting optimal clusterheads and the best routing path.

The rest of the paper is organized as the following. Section 2 includes a detailed survey of the related work. Section 3 introduces Multi-Criteria Decision Making Approaches. Section 4 describes the proposed cluster based context-aware routing protocol (CBCA). Section 5 presents simulation and analysis of the experimental results. Finally, Section 6 concludes the paper.

\section{RELATED WORK}

Selecting the best clusterhead and discovering the efficient routing path are very important to achieve a high accuracy and reliable network in the mobile environments. So, many researchers have been worked to fulfill this purpose and many protocols have been introduced. In this section, we will review in briefly the previous proposed routing protocols schemes in mobile environments.

\section{A. Routing Protocols in Mobile Environments}

Routing is the process of transferring the packets between the networks or within the network from the source to the destination node. Routing is mainly done by specially configured nodes which are called routers and is often confused with the bridging techniques. By basing on network structure, routing methods are categorized as Proactive (Table Driven) Routing Protocols, Reactive (On Demand) Routing Protocols and Hybrid Routing Protocols [1]. In proactive routing such as $D S D V$, each node maintains one or more routing tables. Proactive protocols continuously learn the topology of the network by exchanging topological information among the network nodes. The differences among the protocols lie in their routing table structure, number of tables, updating frequency, use of control messages and the presence of a central node. In Reactive routing protocol such as $A O D V, D S R$ and TORA routes from source to destination doesn't exist. Whenever route is required each node discovers and maintains the route as and when required. In On-Demand routing protocol paths are explored only when needed. Hybrid routing protocols such as ZRP include the features of proactive and reactive routing protocols. Proactive tactic is used to discover and maintain routes to nearer nodes, while routes for far away nodes are discovered reactively. The author in [1] introduced a survey of routing algorithms for mobile networks.

Ad hoc On-demand Distance Vector Routing (AODV) [2] is very popular routing protocol that is based on classical distance vector routing algorithm. AODV is essentially a combination of both DSR [3] and DSDV [4]. It shares DSR's on-demand characteristics hence discovers routes whenever it is needed via a similar route discovery process. However, AODV is loop-free due to the destination sequence numbers associated with routes. It creates routes only when they are needed, which reduces the periodic control message overhead which is associated with proactive routing protocols. AODV adapts traditional routing tables, one entry per destination which is in contrast to DSR that maintains multiple route cache entries for each destination. The initial design of AODV is undertaken after the experience with DSDV routing algorithm. AODV also has other significant features. Whenever a route is available from source to destination, it does not add any overhead to the packets. However, route discovery process is only initiated when routes are not used and/or they expired and consequently discarded. This strategy reduces the effects of stale routes as well as the need for route maintenance for unused routes. AODV have the ability to provide unicast, multicast and broadcast communication. AODV uses a broadcast route discovery algorithm and uses the unicast in route reply massage.

\section{B. Cluster Based Routing Protocols}

Mobile networks are characterized as dynamic topology, bandwidth and link capacity, nodes are energy constrained. Network cluster methods allow fast connection and topology management, better routing and also improve network lifetime, routing delay, bandwidth consumption and throughput. Each cluster in the network contains clusterhead $(\mathrm{CH})$. The $\mathrm{CH}$ responsible to provide communication bridge between members and the other clusters. In the mobile environments, the topology changes dynamically. So, to achieve a high performance in the network, any clustering algorithm should operate with minimum overhead of cluster maintenance and try to preserve its structure as much as possible when nodes are moving and/or the topology is slowly changing. Many approaches for network clustering have been developed by researchers which focus on different performance metrics, most used weight metrics like average consumed power, residual energy, computing capabilities, distance with all neighbors, mobility and node trust value. Most of previous clustering approaches focus on some of this metrics to evaluate the network node. In clustered network each $\mathrm{CH}$ is responsible for the following jobs:

- Identify each node in the cluster (assign IDs).

- Calculate the path weight (cost) of sending/receiving data to all neighbor clusters.

- Communicate all mobile nodes in the cluster

- Define the path that will receive data from across the gateways, and report its gateway which is located on this path to receive data from the common cluster.

- Define routing table.

- Communicate with other clusters through the gateways.

- Send data to all cluster's member nodes.

Naeimi et. al. [5] has introduced taxonomy of $\mathrm{CH}$ selection. In this survey, the clusterhead selection is classified into selforganized schemes, assisted schemes and multi-factor evaluation schemes. Cluster based routing is a most convenient way to develop an efficient routing scheme in mobile environments. But it has to deal with several problems like, control overhead of cluster formation, maintenance, battery Power, stability of cluster, fairness, load balancing etc. So, authors in [6]. Summarize that to optimize the clusterhead election algorithm and to perform efficient cluster based routing in mobile environments, it is necessary to consider all metrics rather than focusing on particular metric. 
Using clustering method in mobile network gives the network several advantages. These advantages introduce them as the most compatible routing protocols in these environments. We list some of these advantages as the following:

- Minimizing the total transmission energy.

- Balancing the energy-exhausting load among all nodes.

- Reducing the bandwidth demand and efficient use of limited channel bandwidth.

- Eliminating the redundant and highly route discovery process.

- Routing path limited to the clusterhead and gateways and thus generating small-size routing tables.

- Increasing the lifetime and scalability of the network.

To ensure that the selection of clusterhead achieve all network requirements and increase network lifetime. The selection of clusterhead must be a multi-criteria decision issue with complex inter-relation between factors. Barfunga et. al [7], introduced an Energy Efficient Cluster Based Routing Protocol. Also, Anitha et. al [8], proposed an enhanced cluster based routing protocol for mobile nodes, this protocol is aimed to prolonging the lifetime of the sensor networks by balancing the energy consumption of the nodes. Naeimi et. al. [5] has surveyed that there are two clusterhead selections which is based on Multi-Factor Evaluation Schemes, Analytical Hierarchy Process (AHP) [9] and Fuzzy Logic Controller (FLC). The AHP method is characterized by decomposing complex decision of $\mathrm{CH}$ selection into a hierarchy of more easily understood sub-problem using numerical values and the FLC characterized by smooth noise tolerance, adaptive modifiable rules, low cost and complexity, more flexible to variable range of applications. Therefore, using a multi-criteria decision making to evaluate mobile nodes according its context will increase network lifetime and decrease network overhead. In this paper, we will introduce a cluster based routing protocol that utilizes the context as a multi-criteria decision making problem to select the optimum clusterhead and select the best routing path. A detailed survey on cluster based routing protocols can be found in [10], [11], [12], [13].

\section{MUlTI-CRITERIA DECISION MAKING APPROACHES}

\section{A. Fuzzy Sets and Fuzzy Number}

Zadeh (1965) introduced the Fuzzy Set Theory (FST) to deal with the uncertainty and ambiguous of data. A major contribution of FST is the capability of representing uncertain data. FST also allows mathematical operators and programming to be performed to the fuzzy domain. A Fuzzy Set (FS) is a class of objects with a continuum of grades of membership. Such a set is characterized by a membership function, which assigns to each object a grade of membership ranging "between" zero and one.

Fuzzy Set: A fuzzy set $\tilde{A}$. In a universe of discourse $\mathrm{X}$ is characterized by a membership function $\tilde{\mu}_{A}(x)$ which associates with each element $\mathrm{X}$ in $\mathrm{X}$ a real number in the interval $[0,1]$. The function value $\tilde{\mu}_{A}(x)$ is termed the grade of membership of $\mathrm{x}$ in $\tilde{A}$. L.A. Zadeh [14].

Triangular Fuzzy Number: A triangular fuzzy number $\tilde{A}$ can be defined by a triplet (L, M, U) shown in Fig-1. The membership function $\tilde{\mu}_{A}(x)$ is defined in [15] as

$$
\tilde{\mu}_{A}(x)=\left\{\begin{array}{lr}
0 & x<L \\
\frac{x-L}{M-L} & L \leq x \leq M \\
\frac{x-U}{M-U} & M \leq x \leq U \\
1 & x>U
\end{array}\right.
$$

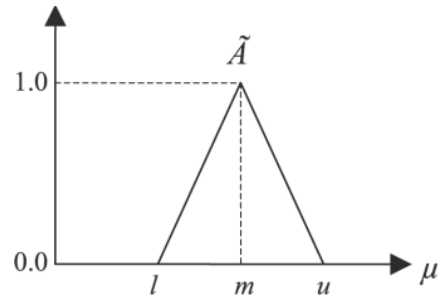

Fig. 1. Triangular Fuzzy Number (L, M, U)

A Symbol " " will be placed above $\mathrm{A}$ if the A shows a FST. A Triangular Fuzzy Number (TFN) A, $\tilde{A}$ TFN represented with three points as follows: (L, M, U). L stand for the lower bound of the fuzzy number, and $U$ stand for the upper bound. This representation is interpreted as membership functions and holds the following conditions.

- L to $\mathrm{M}$ is increasing function

- $\mathrm{M}$ to $\mathrm{U}$ is decreasing function

- $\mathrm{L} \leq \mathrm{M} \leq \mathrm{U}$

Fuzzy sets were originally proposed to deal with problems of subjective uncertainty. Subjective uncertainty results from using linguistic variables to represent the problem or the event, linguistic variable is a variable that is expressed by verbal words or sentences in a natural or artificial language. Linguistic variables are also employed as a way to measure the achievement of the performance value for each criterion. Since the linguistic variables can be defined by the corresponding membership function and the fuzzy interval. Linguistic variables were proposed in [16], For example, linguistic variables with triangular fuzzy numbers may take on effect values such as very high (very good), high (good), fair, low (bad), and very low (very bad). So, we can naturally manipulate the fuzzy numbers to deal with the FMADM problems. The membership function of linguistic variables represented in triangular fuzzy number showed in Fig-2.

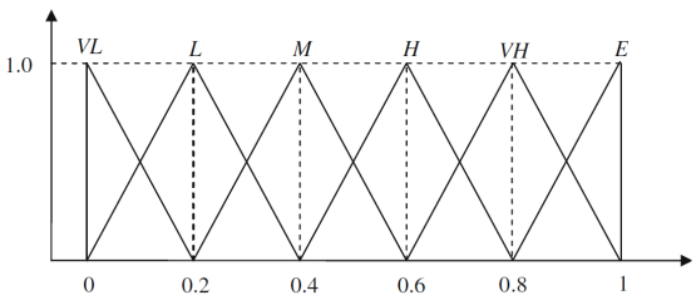

Fig. 2. Triangular fuzzy numbers of linguistic variables 


\section{B. Analytic Hierarchy Process (AHP)}

Bernoulli (1738) proposed the concept of utility function to reflect human persuit, such as maximum satisfaction, and von Neumann and Morgenstern (1947) presented the theory of game and economic behavior model, which expanded the studies on human economic behavior for multiple criteria decision making (MCDM) problems [16], an increasing amount of literature has been engaged in this field. The MCDM can be summarized in five main steps as follows:

1) Define the nature of the problem.

2) Construct a hierarchy system for its evaluation Fig-3.

3) Select the appropriate evaluation model.

4) Obtain the relative weights and performance score of each attribute with respect to each alternative.

5) Determine the best alternative according to the synthetic utility values, which are the aggregation value of relative weights, and performance scores corresponding to alternatives.

6) Outrank the alternatives referring to their synthetic fuzzy utility values from Step 5.

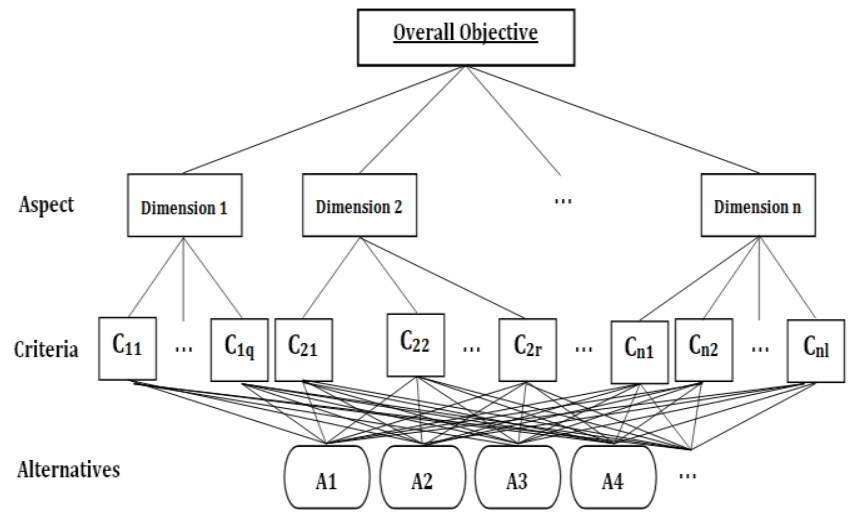

Fig. 3. Hierarchical system for MADM

The analytic hierarchy process $(A H P)$ was proposed to derive the relative weights according to the appropriate hierarchical system. There are four methods, including the eigenvalue method, the geometric mean method, the linear programming method and the lambda-max method to derive the weights using the $A H P$. Only the eigenvalue method is employed to deal with crisp numbers and the other methods are adapted to handle the $A H P$ under fuzzy numbers [16].

In $A H P$ method, the pairwise comparisons for each level with respect to the goal of mobile evaluation are conducted using a nine-point scale. Each pairwise comparison represents an estimate of the priorities of the compared context attribute. The nine-point scale developed by Saaty (1980). Table I expresses preferences between options as equally, moderately, strongly, very strongly, or extremely preferred. These preferences are translated into pairwise weights of 1, 3, 5, 7, and 9, respectively, with $2,4,6$, and 8 as intermediate values.
TABLE I. RATIO SCALE IN AHP (SAATY (1980))

\begin{tabular}{|c|c|c|}
\hline Linguistic & $\begin{array}{c}\text { Intensity } \\
\text { Importance }\end{array}$ & Description \\
\hline $\begin{array}{l}\text { Equally } \\
\text { important }\end{array}$ & 1 & $\begin{array}{l}\text { Two factors contribute equally to } \\
\text { the objective }\end{array}$ \\
\hline $\begin{array}{l}\text { Moderately } \\
\text { more important }\end{array}$ & 3 & $\begin{array}{l}\text { Experience and judgment slightly } \\
\text { favor one over the other }\end{array}$ \\
\hline $\begin{array}{l}\text { Strongly more } \\
\text { important }\end{array}$ & 5 & $\begin{array}{l}\text { Experience and judgment strongly } \\
\text { favor one over the other }\end{array}$ \\
\hline $\begin{array}{l}\text { Very strongly } \\
\text { more important }\end{array}$ & 7 & $\begin{array}{l}\text { Experience and judgment very } \\
\text { strongly favor one over the other. } \\
\text { Its importance is demonstrated in } \\
\text { practice }\end{array}$ \\
\hline $\begin{array}{l}\text { Extremely more } \\
\text { important }\end{array}$ & 9 & $\begin{array}{l}\text { The evidence favoring one over } \\
\text { the other is of the highest possible } \\
\text { validity }\end{array}$ \\
\hline $\begin{array}{l}\text { Intermediate } \\
\text { values }\end{array}$ & $2,4,6,8$ & When compromise is needed \\
\hline
\end{tabular}

\section{Fuzzy AHP}

The global weights for each candidate is determined and the candidates fuzzy priorities are calculated based on subfactors using Linguistic variables, which are defined for the triangular fuzzy numbers, see Table II:

TABLE II. LINGUISTIC VALUES AND FUZZY NUMBERS

\begin{tabular}{|c|c|}
\hline Linguistic values & Fuzzy numbers \\
\hline Very low (VL) & $(0,0,0.2)$ \\
\hline Low (L) & $(0,0.2,0.4)$ \\
\hline Medium (M) & $(0.2,0.4,0.6)$ \\
\hline High (H) & $(0.4,0.6,0.8)$ \\
\hline Very high (VH) & $(0.6,0.8,1)$ \\
\hline Excellent (E) & $(0.8,1,1)$ \\
\hline
\end{tabular}

The geometric mean method was first employed by Buckley (1985) to extend the $A H P$ to consider the situation of using linguistic variables (Zadeh 1965). The degrees of the pairwise comparison of linguistic variables can be expressed using the fuzzy numbers see the following table. Table III.

TABLE III. THE PAIRWISE COMPARISON OF LINGUISTIC VARIABLES USING FUZZY

\begin{tabular}{|c|c|c|}
\hline Intensity of fuzzy scale & Fuzzy numbers & Number user defined \\
\hline$\tilde{1}$ & $(\mathrm{~L}, \mathrm{M}, \mathrm{U})$ & $(\ldots, 1, \ldots)$ \\
\hline$\tilde{3}$ & $(\mathrm{~L}, \mathrm{M}, \mathrm{U})$ & $(\ldots, 3,3)$ \\
\hline$\tilde{5}$ & $(\mathrm{~L}, \mathrm{M}, \mathrm{U})$ & $\left(\ldots, 5,{ }_{-}\right)$ \\
\hline$\tilde{7}$ & $(\mathrm{~L}, \mathrm{M}, \mathrm{U})$ & $\left(,, 7,{ }_{-}\right)$ \\
\hline$\tilde{9}$ & $(\mathrm{~L}, \mathrm{M}, \mathrm{U})$ & $\left(\ldots, 9,{ }_{-}\right)$ \\
\hline$\tilde{2}, \tilde{4}, \tilde{6}, \tilde{8}$ & $(\mathrm{~L}, \mathrm{M}, \mathrm{U})$ & $\left({ }_{-},{ }_{-}\right)$ \\
\hline
\end{tabular}

From the information of the pairwise comparison, we can form the fuzzy positive reciprocal matrix as the following:

$$
\tilde{A}=\left[\begin{array}{cccc}
a_{11} & a_{12} & \cdots & a_{1 n} \\
a_{21} & a_{22} & \cdots & a_{2 n} \\
\vdots & \vdots & \vdots & \vdots \\
a_{n 1} & a_{n 2} & \cdots & a_{n n}
\end{array}\right]
$$


Where $\tilde{a}_{i j} \odot \tilde{a}_{j i} \approx 1$ and $\tilde{a}_{i j} \cong \frac{w_{i}}{w_{j}}$

Then, the geometric mean method for finding the final fuzzy weights of each criterion can be formulated as the following:

$$
\widetilde{w}_{i}=\tilde{r}_{i}\left(\tilde{r}_{1} \oplus \tilde{r}_{2} \oplus \ldots \oplus \tilde{r}_{n}\right)^{-1}
$$

Where

$$
\tilde{r}_{i}=\left(\tilde{a}_{i 1} \odot \tilde{a}_{i 2} \odot \ldots \odot \tilde{a}_{i n}\right)^{1 / n}
$$

The fuzzy weights of each criterion can also be defuzzified by center of area (CoA) in order to obtain a crisp solution.

\section{TOPSIS}

The Technique for Order Preferences by Similarity to an Ideal Solution (TOPSIS) method was proposed by Hwang and Yoon (1981). The main idea came from the concept of the compromise solution to choose the best alternative which has the shortest Euclidean distance from the positive ideal solution (optimal solution) and farthest Euclidean distance from the negative ideal solution. The positive-ideal solution (PIS) is a solution that maximizes the benefit criteria and minimizes the cost criteria, whereas the negative ideal solution (NIS) maximizes the cost criteria and minimizes the benefit criteria. Then, choose the best one of sorting, which will be the best alternative. So, according to this technique we can evaluate mobile node based on the context.

\section{E. Fuzzy TOPSIS}

The use of numerical values (Crisp values) in the rating of alternatives may have limitations to deal with uncertainties and ambiguous. So, extensions of TOPSIS were developed to solve problems of decision making with uncertain data resulting in fuzzy TOPSIS. In practical applications, the triangular shape of the membership function is often used to represent fuzzy numbers. Fuzzy models using triangular fuzzy numbers proved to be very effective for solving decision-making problems where the available information is imprecise.

Given a set of alternatives, $A=\left\{A_{k} \mid k=1, \ldots, n\right\}$, and a set of criteria, $C=\left\{C_{j} \mid j=1, \ldots, m\right\}$, where $X=\left\{X_{k j} \mid k=1, \ldots, n\right.$; $j=1, \ldots, m\}$ denotes the set of performance ratings and $w=$ $\left\{w_{j} \mid j=1, \ldots, m\right\}$ is the set of weights, the information table $I$ $=(A, C, X, W)$ can be represented as shown in TableIV. The first step of TOPSIS is to calculate normalized ratings by TableIV.

TABLE IV. TOPSIS INFORMATION TABLE I $=(\mathrm{A}, \mathrm{C}, \mathrm{X}, \mathrm{W})$

\begin{tabular}{|ccccc|}
\hline Alternatives & $\mathrm{C}_{1}$ & $\mathrm{C}_{2}$ & $\cdots$ & $\mathrm{c}_{\mathrm{m}}$ \\
$\mathrm{A} 1$ & $\mathrm{x}_{11}$ & $\mathrm{x}_{12}$ & $\cdots$ & $\mathrm{x}_{1 \mathrm{~m}}$ \\
$\mathrm{~A} 2$ & $\mathrm{x}_{21}$ & $\mathrm{x}_{22}$ & $\cdots$ & $\mathrm{x}_{2 \mathrm{~m}}$ \\
$\vdots$ & $\vdots$ & $\vdots$ & $\vdots$ & $\vdots$ \\
An & $\mathrm{a}_{\mathrm{n} 1}$ & $\mathrm{a}_{\mathrm{n} 2}$ & $\cdots$ & $\mathrm{a}_{\mathrm{nn}}$ \\
$\mathrm{w}$ & $\mathrm{w}_{1}$ & $\mathrm{w}_{2}$ & $\cdots$ & $\mathrm{w}_{\mathrm{m}}$ \\
\hline
\end{tabular}

Now we will list the TOPSIS main steps as the following:

Step 1: Construct the normalized decision matrix

To transform the various attribute dimensions into nondimensional attributes, which allows comparison across the attributes. The first step of TOPSIS is to calculate normalized ratings using the following equation:

$$
r_{k j}(x)=\frac{x_{k j}}{\sqrt{\sum_{k=1}^{n} x_{k j}^{2}}} \mid k=1, \ldots, n ; j=1, \ldots, m ;
$$

Step 2: Construct the weighted normalized decision matrix

- For benefit criteria (larger is better)

$$
r_{k j}(x)=\frac{x_{k j}-x_{j}^{-}}{x_{j}^{*}-x_{j}^{-}}
$$

Where $x_{j}^{*}=\max _{k} x_{k j}$ and $x_{j}^{-}=\min _{k} x_{k j}$ or setting $x^{*}$ is the aspired/desired level and $x$ is the worst level.

- For cost criteria (smaller is better)

- $r_{k j}(x)=\frac{x_{j}^{-}-x_{k j}}{x_{j}^{-}-x_{j}^{*}}$

Step 3: Calculate weighted normalized ratings using

$v_{k j}(x)=w_{j} r_{k j}(x) \mid k=1, \ldots, n ; j=1, \ldots, m$;

Step 4: Obtain the positive ideal point (PIS) and the negative ideal point (NIS)

$A^{+}$represents positive ideal point and $A^{-}$represents negative ideal point.

$$
\begin{aligned}
A^{+}= & \left\{v_{1}^{+}, v_{2}^{+}, \ldots, v_{j}^{+}, \ldots, v_{m}^{+}\right\} \\
= & \left\{\left(\max _{\mathrm{k}} \mathrm{v}_{\mathrm{kj}}(\mathrm{x}), \mathrm{j} \in \mathrm{J}_{1}\right),\left(\min _{\mathrm{k}} \mathrm{v}_{\mathrm{kj}}(\mathrm{x}), \mathrm{j} \in \mathrm{J}_{2}\right)\right. \\
& \mid, \mathrm{k}=1, \ldots, \mathrm{n} ;\} \\
A^{-}= & \left\{v_{1}^{-}, v_{2}^{-}, \ldots, v_{j}^{-}, \ldots, v_{m}^{-}\right\} \\
= & \left\{\left(\min _{\mathrm{k}} \mathrm{v}_{\mathrm{kj}}(\mathrm{x}), \mathrm{j} \in \mathrm{J}_{1}\right),\left(\max _{\mathrm{k}} \mathrm{v}_{\mathrm{kj}}(\mathrm{x}), \mathrm{j} \in \mathrm{J}_{2}\right)\right. \\
& \mid, \mathrm{k}=1, \ldots, \mathrm{n} ;\}
\end{aligned}
$$

Where $J_{1}$ and $J_{2}$ are the benefit and the cost attributes, respectively

Step 5: Calculate the separation from the PIS and the NIS between alternatives.

The separation values can be measured using the Euclidean distance, which is given as: $D_{k}^{*}$, Positive Ideal Separation and $D_{k}^{-}$negative Ideal Separation.

$$
\begin{aligned}
& D_{k}^{*}=\sqrt{\sum_{j=1}^{m}\left[v_{k j}(x)-v_{j}^{+}\right]^{2}}, \quad k=1, \ldots, n ; \\
& D_{k}^{-}=\sqrt{\sum_{j=1}^{m}\left[v_{k j}(x)-v_{j}^{-}\right]^{2},} \quad k=1, \ldots, n ;
\end{aligned}
$$

Step 6: Calculate the Relative Closeness to the Ideal Solution

$$
\begin{aligned}
& C_{k}^{*}=\frac{D_{k}^{-}}{D_{k}^{*}+D_{k}^{-}} \\
& \text {Where } C_{k}^{*} \in[0,1] \quad \forall \mid \mathrm{k}=1, \ldots, \mathrm{n} ;
\end{aligned}
$$

Finally, the preferred orders can be obtained according to the similarities to the PIS $\left(C_{k}^{*}\right)$ in descending order to choose the best alternatives. 


\section{Proposed Cluster Based Routing PROTOCOL}

The proposed model of clusterhead selection problem combines of two MCDM approaches FAHP and FTOPSIS approaches to evaluate mobile node which is based on different context attributes as mobility, available bandwidth, residual energy and number of neighbors. The main difference between our proposed clusterhead selection method and other clustering algorithms that other methods rely on only one or two factors to complete the clustering process which is not sufficient to increase network lifetime and solve network overhead problem. Also, these methods ignore other factors which affect the network lifetime. This section will illustrate in details the new cluster based context-aware (CBCA) routing protocol. So, we offer a detailed explanation of the new protocol as the following.

\section{A. Setup Phase}

Setup phase consists of three levels to obtain the final results of ranking mobile nodes. In the first level: The Contextaware Middleware responsible for collecting the contextual information that will represent the criteria. The second level: Obtain the best weight for each criteria using FAHP. Finally, the third level: Responsible for using FTOPSIS to evaluate the alternatives and determine the final mobile node rank value.

Each mobile node collects the needed context for the evaluation operation. According to Gad-ElRab [17], the context-aware middleware is committed to support each mobile node by the contextual information which is needed in this operation. The context-aware middleware will retrieve context from its source and assign a new value to the mobile node. The source node broadcasts a hello message to all nodes in the network to inform each node to share its context with other nodes. This message contains a specified context attributes (mobility value, bandwidth, energy level and number of its neighbor nodes). These attributes will be used to evaluate each mobile node. All nodes that receive the hello message from source node will replay and send its context values to the source node or any other specified node in the network.

Now it's a time to start Device Dependent Context Rank $(D D C R)$ operation. From [17], Device Dependent Context represents any contextual information that characterizes the device such as processing capabilities, energy level, available bandwidth, input sensors, visualization capabilities etc. So, each node will be evaluated based on its device dependent context using FAHP and FTOPSIS approaches. The FAHP method will compute the weight of each context attribute which will contribute in computing $D D C R$, note that computing weight is a pre-calculated by the application for one time only at the first of establishing the network. Fig-4 illustrates the decision hierarchy of mobile nodes evaluation process. The FTOPSIS will compute the final evaluation value of each node based on weight value from FAHP method and the received context from all nodes in the network. The source starts to normalize rating values which are received from each node and calculates weighted normalized ratings for all criteria. Then, it calculates positive and negative ideal point (A+, A-). After that, the source node will determine Positive Ideal
Separation and Negative Ideal Separation D+, D- for each node. Finally, it will determine the global context evaluation. At this point, the source obtains a rank value for each node in the network. After that, the source node broadcasts a message to inform each node by its own rank value. Then cluster formation phase will start.

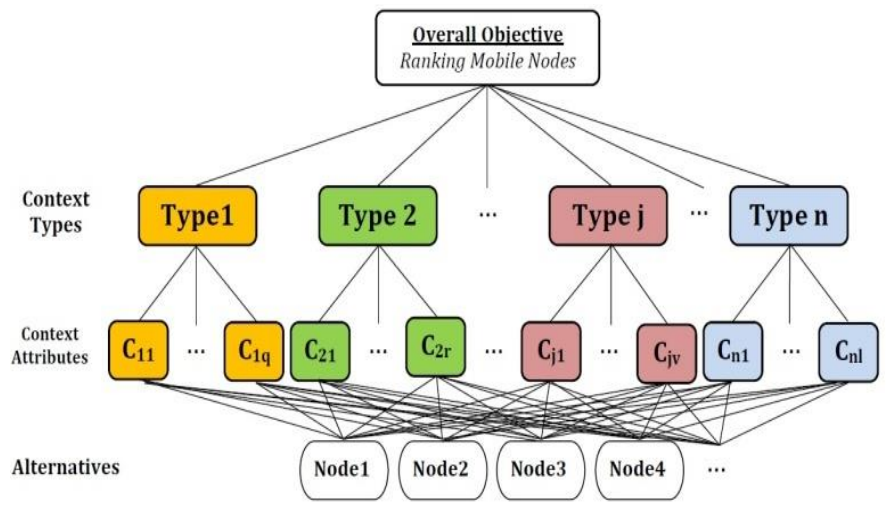

Fig. 4. Hierarchical system for Mobile Nodes Ranking

\section{B. Cluster Formation Phase}

In cluster formation phase, the network is partitioned into a number of clusters, each cluster contains one clusterhead $\mathrm{CH}$. The $C H$ is a node which has the largest $D D C R$ value in the cluster, the remaining nodes in the cluster called member nodes. To start cluster formation phase, we have a number on evaluated nodes and each one knows its $D D C R$ value, each node will start to check if any of its neighbor have rank value larger than its rank. If there is one or more; then the node will choose the largest of them and this selected node will be a parent of this node, and this node will be a member node in this cluster. If a node has the largest $D D C R$ among all neighbors, this node will become $\mathrm{CH}$ of its cluster. Each selected $\mathrm{CH}$ sends an advertisement message to all nodes that belong to the cluster, this message is a small message containing the $\mathrm{CH}$ ID and the IDs of all nodes in its cluster. If there is a node has the largest $D D C R$ from all its neighbor and does not select as a $\mathrm{CH}$; then this node will select the nearest and the largest neighbor to be its parent. Until now, we have a number of clusters and each cluster contains one node which is called $\mathrm{CH}$, this $\mathrm{CH}$ has the largest $\mathrm{DDCR}$ in the cluster and each cluster consists of $K$ hops. A new problem arises after forming clusters which is draining of $\mathrm{CH}$ resources. The $\mathrm{CH}$ is responsible for many jobs in its cluster, these jobs consume the $\mathrm{CH}$ resources. So, to decrease this drains of $\mathrm{CH}$ s, each $\mathrm{CH}$ will elect number of nodes in its cluster to help it to accomplish some jobs such as communicating with other clusters, do some calculations and data collection, etc. The selected nodes are called Vice $\mathrm{CH}$ and any Vice $\mathrm{CH}$ falls on the first hop of its $\mathrm{CH}$, and has the highest $D D C R$ between neighbor, has the lowest connectivity, does not belong to any path to source node or other $\mathrm{CHs}$. The $\mathrm{CH}$ of any cluster may have a Vice $\mathrm{CH}$ or not. The gateway nodes of any cluster will be authorized from the $\mathrm{CH}$ by handling any operation from any other clusters, such as calculating path weight between two clusters or other jobs. Fig5 illustrates the cluster formation phase. 


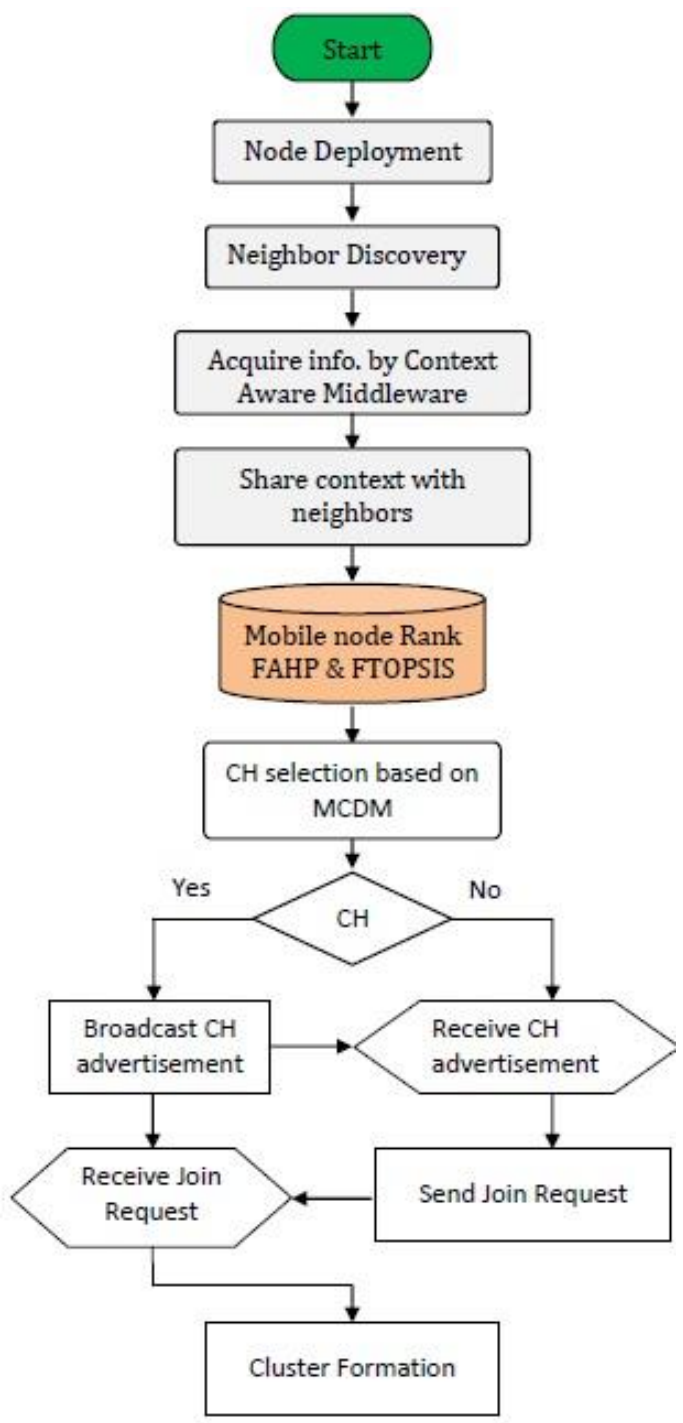

Fig. 5. Flow Diagram of Proposed Clustring Method

\section{Cluster Maintenance Phase}

Cluster maintenance phase will solve the problem of load balancing in network clusters. Each $\mathrm{CH}$ will calculate the average $D D C R$ of its cluster. If the average $D D C R$ is smaller than any of its neighbor clusters, then the cluster will discard the extra low DDCR nodes for the benefit of the largest neighbor cluster if it's possible. In the discrediting operation, each common node in the cluster knows the average $D D C R$ value and the average $D D C R$ value of its neighbor clusters; then the common node will join to the largest average $D D C R$ value of its neighbor if its $D D C R$ is very small.

If any member node separated from its cluster, it will attend to the nearest and the largest $D D C R$ neighbor cluster. If the gateway node separated from its cluster, the $\mathrm{CH}$ will change its receiving path using the presented scenario in the previous discussion. If the separated node is a $\mathrm{CH}$, its member nodes will try to join to the nearest cluster using the proposed scenario. If a new node (unranked node) adds to the network, it will join to the nearest cluster without computing $D D C R$.

\section{Determining Cluster's Getaways}

The gateway node is defined as a common node between two different clusters, if there is a routing path between two clusters, the sent message will pass through the common node that is called gateway. Each gateway knows the number of nodes in each common cluster and all paths to the common clusters. The gateway that is common to more than two clusters is worthies to handle communication of the common clusters. If the gateway node is connected to more than one node in the other cluster, it will select the highest $D D C R$ and nearest gateway to make a path to the other $\mathrm{CH}$. The $\mathrm{CH}$ is worthier to send the message to other cluster if it was directly connected to another cluster (the node is $\mathrm{CH}$ and gateway at the same time). If there is more than one gateway to the same cluster, the $\mathrm{CH}$ will select the largest $D D C R$ node to be a gateway. Each $C H$ sends data first to all used gateways after finishing the $\mathrm{CH}$ distributes data to all member nodes which sorted according to $D D C R$ values.

\section{E. Discovering Routing Path}

In this phase, the network will discover the best routing path from source to destination, determining routing path begins by computing all paths weights (cost) between source node and destination node. The routing path cost will be derived from the average $D D C R$ of each path $A_{r}$ and its number of hops (Delay cost) $D$, as the following:

$$
R_{c_{i}}=\frac{1}{D_{i}} w_{1}+A_{r_{i}} w_{2}, i=1, \ldots, p ;
$$

Such that $w_{1}$ represents the weight of delay cost $D, w_{2}$ represents the weight of $\mathrm{A}_{r}$ of each path, and $p$ is the number of discovered paths. The network application will commit to determine the values of $w_{1}$ and $w_{2}$ such that:

$$
w_{1}+w_{2}=1
$$

In this paper we use values of $w_{1}$ and $w_{2}$ as, $w_{1}=0.5$ and $w_{2}$ $=0.5$. The routing path that has maximum $R_{c}$ will be selected to be the path between source and destination nodes.

\section{SIMULATION RESUlTS}

In this section, we present the simulation results of the comparison between the proposed cluster based context-aware CBCA routing protocol and the standard routing protocol $A O D V$ [2]. We implemented the proposed protocol using the OMNET++ simulator [18].

\section{A. Performance Metrics}

We used many ways to study the proposed algorithm. The performance of CBCA protocol evaluated according to the following metrics:

1) Average Packet Delivery Ratio: It is the ratio of the number of successfully received packets to the total number of packets sent.

2) Average end-to-end delay: The end-to-end delay is averaged over all surviving data packets from the sources to the destinations.

3) Control overhead: The control overhead is defined as the total number of routing control packets normalized by the total number of received data packets. 
Due to continuous changes in the topology of the mobile network. We generated different network scenarios for number of nodes, bandwidth and number of messages. Also, we used Random Waypoint mobility to model a mobility of nodes. Table V shows our simulation parameters.

TABLE V. SimUlation PARAMETERS

\begin{tabular}{|c|c|}
\hline Parameter & Value \\
\hline Network Area & $1000 \mathrm{~m} \times 1000 \mathrm{~m}$ \\
\hline Number of Nodes & $25-250$ \\
\hline Initial Energy & $0.5 \mathrm{~J}$ \\
\hline Mobility Type & RandomWPobility \\
\hline Radio Transmission Range & $250 \mathrm{~m}$ \\
\hline Bandwidth & $3-30 \mathrm{Kbps}$ \\
\hline
\end{tabular}

As we mentioned in section 3 , we can obtain the weight value of each criteria using FAHP method. This can be done through pairwise comparisons by asking how much the importance of a criterion compared to another criterion. By using this method, we can deduce all required weight values. So, a mobility weight was 0.074 , bandwidth weight was 0.486 , energy weight was 0.324 and connection number weight was 0.191. The simulation will use these values to obtain final mobile nodes rank value.

\section{B. Simulation Results and Analysis}

In this section, we will discuss the routing protocol simulation results and compare the proposed CBCA protocol and $A O D V$ protocol based on the above mentioned performance metrics.

Fig-6, compares the percentage of packet delivery ratio (PDR) for CBCA and AODV. As shown in Fig-6 PDR decreases as the number of nodes increases. We can see that the packet delivery ratio of CBCA protocol is clearly higher than the AODV protocol and our algorithm can scale up to larger network.

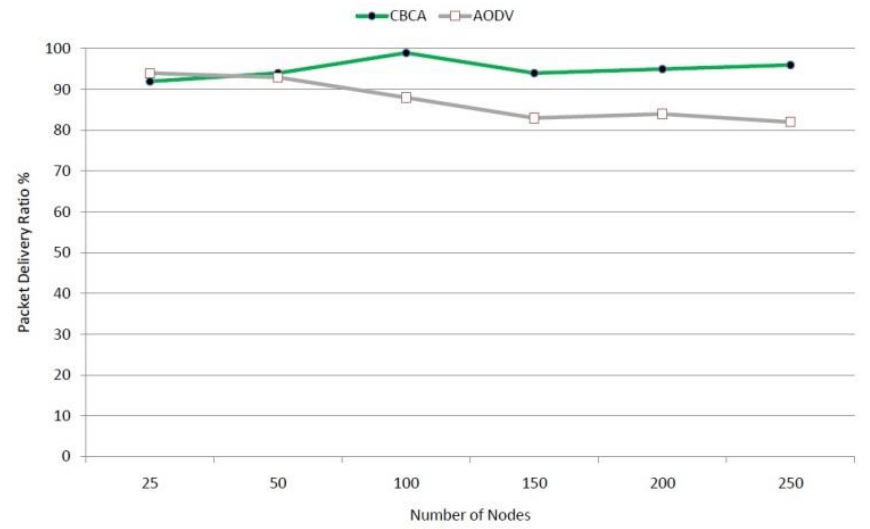

Fig. 6. Packet Delivery Ratio vs. Number of Nodes

The comparison of the end-to-end delay is shown in Fig-7. We can see that as the number of nodes increases, the average end-to-end delay increases, because more connections and congestions appear in higher density network. It can also be concluded that the average end-to-end delay for CBCA protocol is better than the AODV protocol.

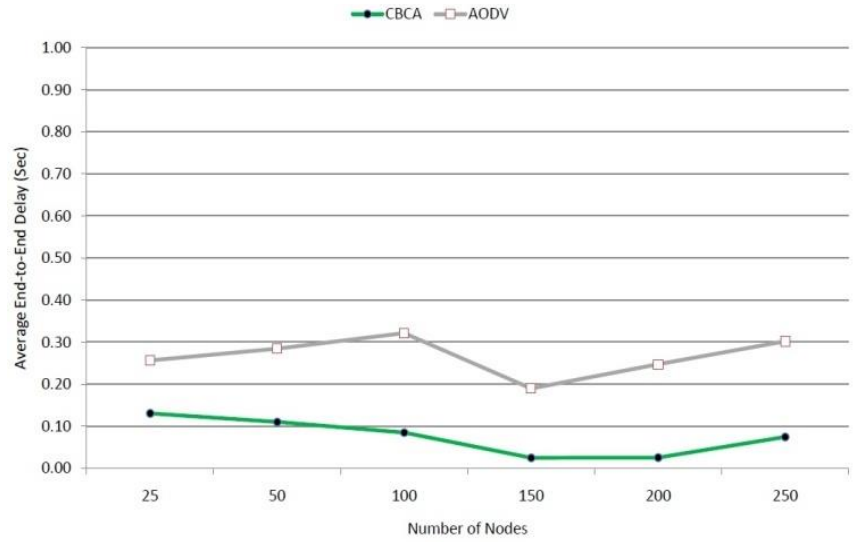

Fig. 7. Average End-to-End Delay vs. Number of Nodes

As shown in Fig-6 and Fig-7, the CBCA protocol enhances the PDR and end-to-end delay because in the CBCA, the route discovery phase and routing packets based on the high performance nodes in the network (the $\mathrm{CHs}$ and the Gateways). The CHs and the Gateways work with higher bandwidth and they have high number of connection in the network. These features minimize the delivery time from source node to destination and maximize packets delivery ratio in all network.

Fig-8, compares the packets overhead for CBCA and AODV. As shown in Fig-8, the packets overhead increases as the number of nodes increases. Also, we can ensure that the control overhead is less for CBCA when it is compared to AODV. So, CBCA protocol is more efficient in larger network.

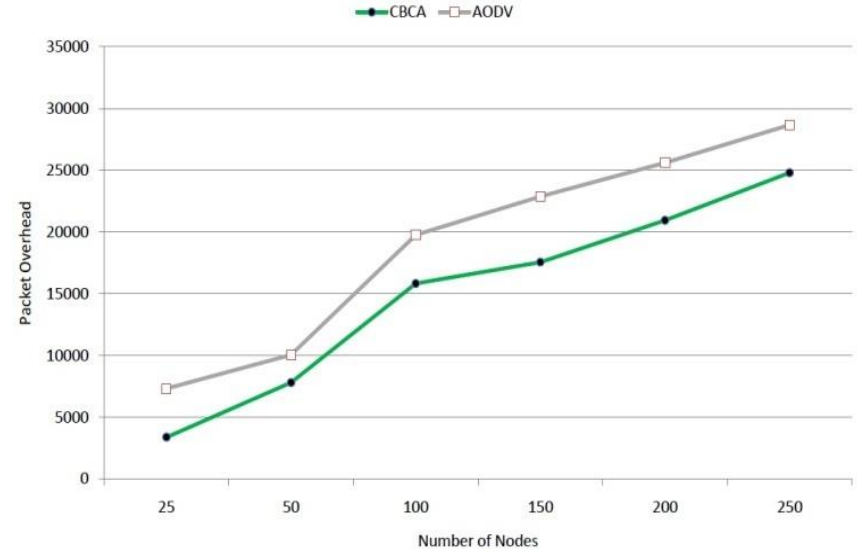

Fig. 8. Packet Overhead vs. Number of Nodes

Fig-9 shows the comparison of packet delivery ratio for CBCA and AODV in different bandwidth. As shown in Fig-9 PDR increases as the bandwidth of nodes increases. So, the number of packet drops also decreases. However, the proposed CBCA protocol achieves good delivery ratio, compared to AODV, which means that our approach has better performance. 


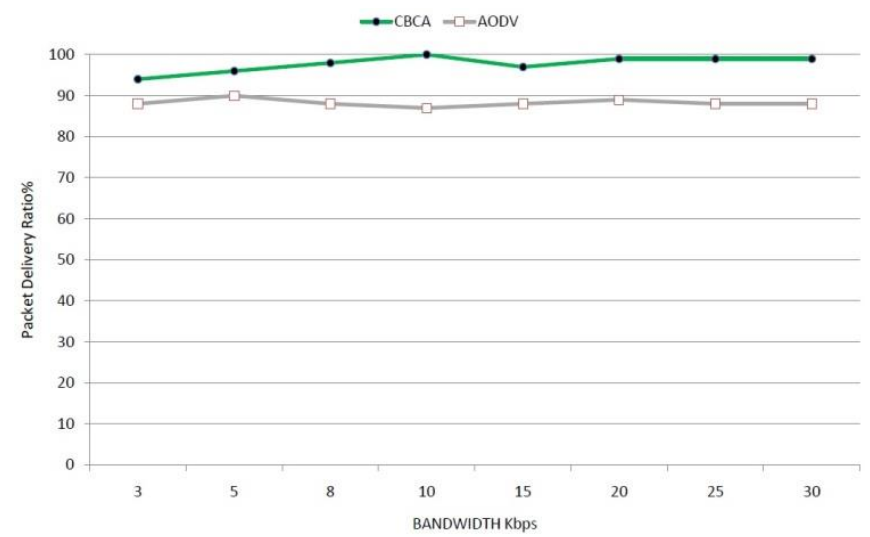

Fig. 9. Packet Delivery Ratio vs. Node Bandwidth

As shown in Fig-10, the average end-to end delay of the proposed CBCA protocol is lower than the AODV protocol. This is because CBCA routing protocol takes into account node bandwidth and it needs smaller route discovery time than AODV.

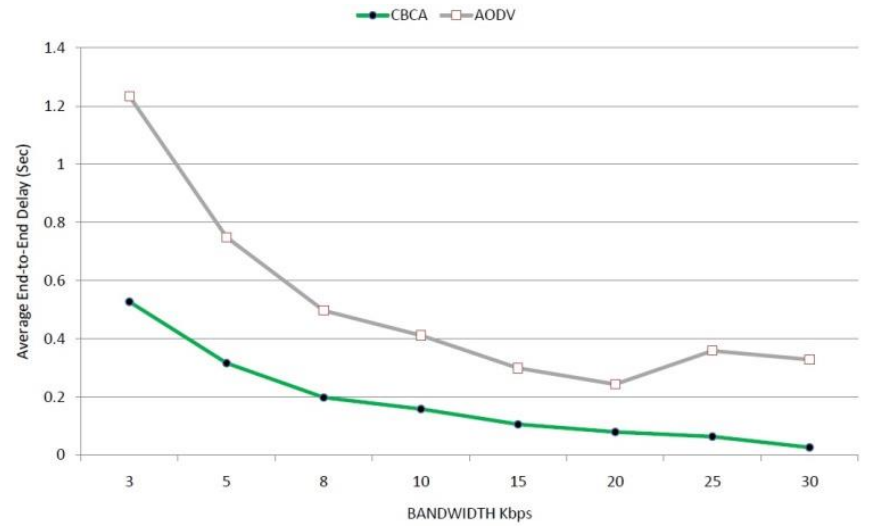

Fig. 10. Average End-to-End Delay vs. Node Bandwidth

Fig-11, shows a comparison between Packet Delivery Ratio and Number of Messages. As shown in Fig-11, PDR decreases as the number of messages increases. We can see that the packet delivery ratio of CBCA protocol is clearly higher than the AODV protocol.

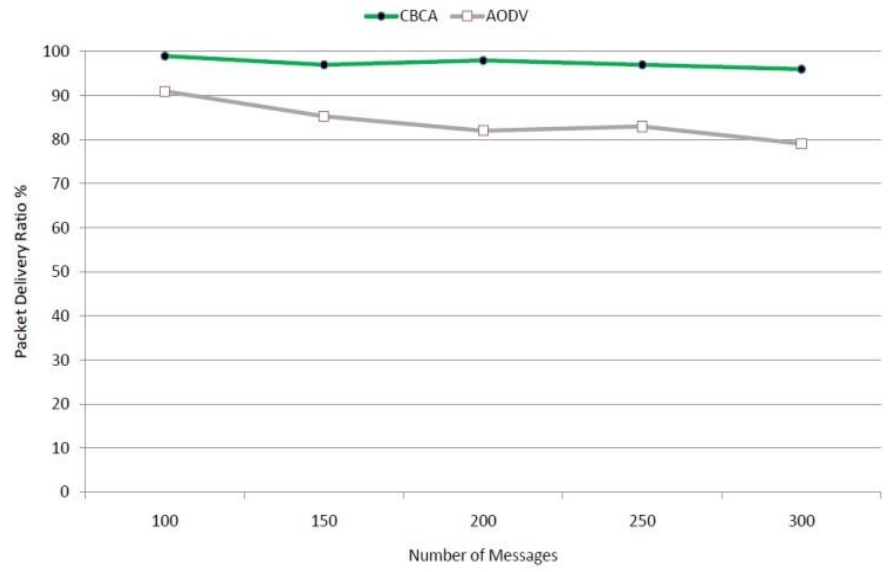

Fig. 11. Packet Delivery Ratio vs. Number of Messages
Fig-12, shows the comparison of the end-to-end delay between CBCA protocol and AODV protocol. We can see that as the number of messages increases, the average end-to-end delay increases. This is because the increasing in the number of messages leads to network congestion, which increases the postponement of sending the messages.

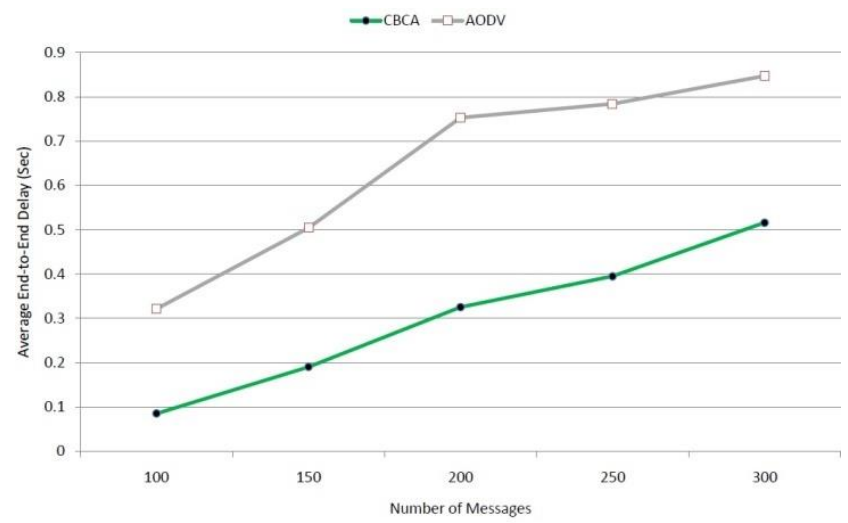

Fig. 12. Average End-to-End Delay vs. Number of Messages

Fig-13 shows the comparison of the packet overhead for CBCA and AODV with various number of messages. For both CBCA and AODV there is increasing in packet overhead with the increases of message number. CBCA routing provides smaller packet overhead than AODV. We can observe that CBCA has small increasing rate. This is because in the cluster formation process, a lot of control packets are exchanged. Also, the proposed CBCA protocol uses small number of nodes in route discovery phase.

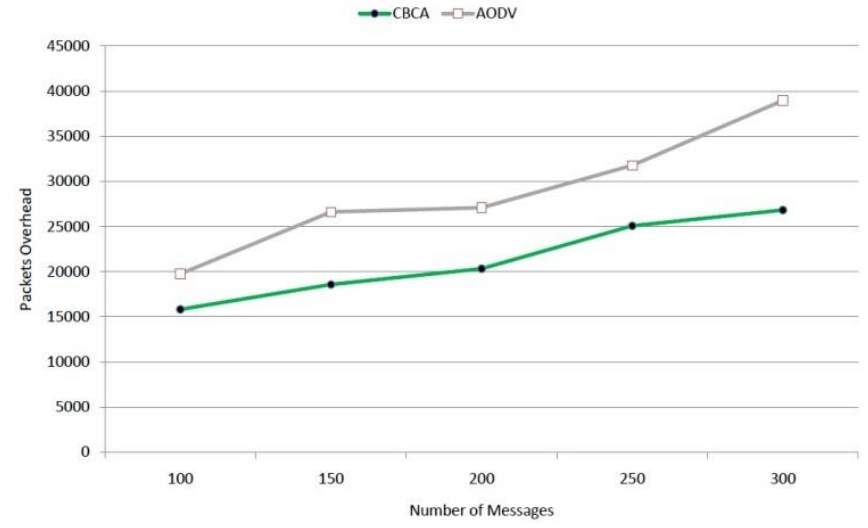

Fig. 13. Packet Overhead vs. Number of Messages

Fig-14 shows the average delay time in different weights of hops number $w_{l}$ as we explained in equation (14) in route path selection process. As shown in this figure, When the weight of hops number was 0.3 to 0.8 given us the best results in average delay time.

\section{CONCLUSION}

In this paper, we introduced the most relevant routing protocols types in mobile environments. Also, we discussed cluster based routing protocols issues. In addition, we proposed a new context based routing protocol in mobile environment. The new CBCA protocol is based on ranking network's nodes 
according to its Device Dependent Context DDCR. The evaluation process is relying on two $M C D M$ approaches

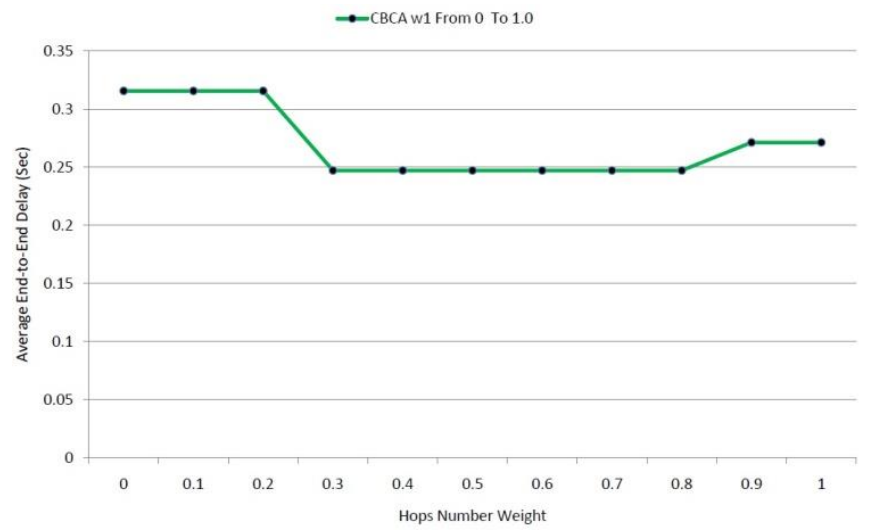

Fig. 14. Average End-to-End Delay vs. Hops Number Weight

FAHP and FTOPSIS to determine the mobile node ranking value. Then, we proposed a network clustering schema based on the previous ranking process. Finally, we introduced a routing discovery model to obtain the optimum routing path between source and destination, the best routing path that has the maximum average rank and minimum hops number. The performance of the new protocol has been evaluated through extensive simulation with various network sizes, bandwidth rate and number of messages. The simulation results demonstrate that there is a significant improvement in packet delivery ratio and the average end to end delay over traditional routing protocol AODV, and better performance than other routing algorithms in literature as well. So, the proposed CBCA protocol can increase the network lifetime and decrease network overhead. Which achieve the reliability and accuracy to the network in these environments.

Our future work involves using CBCA protocol to develop a new adaptive broadcasting protocol in mobile environments, based on user dependent context to reduce the network overhead and deliver the information to the user who will utilize and take care of the shared information.

\section{REFERENCES}

[1] Mamta Dhanda; Shikha Chaudhry. Survey of routing protocols for mobile ad hoc networks. International Journal of Advanced Research in Computer Science and Software Engineering, 3(4):1026-1031, 2013.

[2] Charles E Perkins and Elizabeth M Royer. Ad-hoc on-demand distance vector routing. In Mobile Computing Systems and Applications, 1999. Proceedings. WMCSA'99. Second IEEE Workshop on, pages 90-100. IEEE, 1999.
[3] David B Johnson and David A Maltz. Dynamic source routing in ad hoc wireless networks. In Mobile computing, pages 153-181. Springer, 1996.

[4] Charles E Perkins and Pravin Bhagwat. Highly dynamic destinationsequenced distance-vector routing (dsdv) for mobile computers. In ACM SIGCOMM Computer Communication Review, volume 24, pages 234-244. ACM, 1994.

[5] Soroush Naeimi, Hamidreza Ghafghazi, Chee-Onn Chow, and Hiroshi Ishii. A survey on the taxonomy of cluster-based routing protocols for homogeneous wireless sensor networks. Sensors, 12(6):7350-7409, 2012.

[6] S. Mehta, P. Sharma, and K. Kotecha. A survey on various cluster head election algorithms for manet. In Engineering (NUiCONE), 2011 Nirma University International Conference on, pages 1-6. IEEE, Dec 2011.

[7] S.P. Barfunga, P. Rai, and H.K.D. Sarma. Energy efficient cluster based routing protocol for wireless sensor networks. In Computer and Communication Engineering (ICCCE), 2012 International Conference on, pages 603-607, July 2012

[8] R.U. Anitha and P. Kamalakkannan. Enhanced cluster based routing protocol for mobile nodes in wireless sensor network. In Pattern Recognition, Informatics and Mobile Engineering (PRIME), 2013 International Conference on, pages 187-193, Feb 2013.

[9] Yaoyao Yin, Juwei Shi, Yinong Li, and Ping Zhang. Cluster head selection using analytical hierarchy process for wireless sensor networks. In Personal, Indoor and Mobile Radio Communications, 2006 IEEE 17th International Symposium on, pages 1-5, Sept 2006.

[10] M. Patil and R.C. Biradar. A survey on routing protocols in wireless sensor networks. In Networks (ICON), 2012 18th IEEE International Conference on, pages 86-91, Dec 2012.

[11] D. Sivakumar, B. Suseela, and R. Varadharajan. A survey of routing algorithms for manet. In Advances in Engineering, Science and Management (ICAESM), 2012 International Conference on, pages 625640, March 2012

[12] P. Kumari, M.P. Singh, and P. Kumar. Survey of clustering algorithms using fuzzy logic in wireless sensor network. In Energy Efficient Technologies for Sustainability (ICEETS), 2013 International Conference on, pages 924-928, April 2013.

[13] H. Kiwan and Y.L. Morgan. Hierarchical networks: Routing and clustering (a concise survey). In Electrical and Computer Engineering (CCECE), 2013 26th Annual IEEE Canadian Conference on, pages 1-4, May 2013.

[14] Zadeh Lotfi A. Fuzzy sets. Information and control, 8(3):338-353, 1965.

[15] Kaufmann ; Arnold, Gupta ; Madan M, and Kaufmann ; A. Introduction to fuzzy arithmetic: theory and applications. Van Nostrand Reinhold Company New York, 1985.

[16] G. H Tzeng and Jih-Jeng Huang. Multiple Attribute Decision Making: Methods and Appliations. CRC Press, 2011.

[17] Ahmed. A. A. Gad-ElRab; T. A. A. Alzohairy; Almohammady S. Alsharkawy. Probabilistic context retrieval time-based algorithms in dynamic environments. International Journal of Current Engineering and Technology, 4(3):1633-1642, 2014

[18] OMNET++ OpenSim Ltd. http://www.omnetpp.org. 